\title{
Infant Vocal-Motor Coordination: Precursor to the Gesture-Speech System?
}

\author{
Jana M. Iverson and Mary K. Fagan
}

\begin{abstract}
This study was designed to provide a general picture of infant vocal-motor coordination and test predictions generated by Iverson and Thelen's (1999) model of the development of the gesture-speech system. Forty-seven 6- to 9-month-old infants were videotaped with a primary caregiver during rattle and toy play. Results indicated an age-related increase in frequency of vocal-motor coordination, greater coordination with arm (specifically right arm) than leg or torso movements, and a temporal pattern similar to that in adult gesture-speech coproductions. Rhythmic vocalizations (consonant-vowel repetitions) were more likely to occur with than without rhythmic movement, and with rhythmic manual than with nonmanual activity, and the rate of vocalmanual coordination was higher in babblers than in prebabblers.
\end{abstract}

Speakers in cultures the world over produce spontaneous hand gestures, and these gestures are tightly linked to contemporaneous speech. The strength of this link has been demonstrated in gesture suppression experiments and in studies of communication among blind individuals. Thus, for example, narratives produced with gestures prohibited are more verbally dysfluent than those produced when gesturing is permitted (Rauscher, Krauss, \& Chen, 1996), and congenitally blind speakers, despite never having seen the gestures of others, not only gesture when speaking, but do so even when talking to a blind listener (Iverson, 1999; Iverson \& GoldinMeadow, 1997, 1998, 2001).

One fundamental characteristic of the adult gesture-speech system is that it is temporally coexpressive. When speakers move their hands to add emphasis to particular words and highlight essential phrases, these gestures are highly synchronous with

Jana M. Iverson and Mary K. Fagan, Department of Psychological Sciences, University of Missouri-Columbia. Jana M. Iverson is now at the Department of Psychology, University of Pittsburgh.

Portions of this work were presented at the biennial meetings of the Society for Research in Child Development, Minneapolis, MN, April 2001; the First International Congress on Motor Development and Learning in Infancy, Amsterdam, The Netherlands, June 2001; and the biennial meetings of the Society for Research in Child Development, Tampa, FL, April 2003. The research was supported by grants from the National Institutes of Health (R01 HD41677) and the University of Missouri Research Board to the first author. We thank Jeanine Jesberg, Michelle Powers, Stephanie Rifas, Jennifer Rust, and Tracy Warnecke for assistance with data collection, coding, and establishing reliability; Esther Thelen and Robert Wozniak for their intellectual contributions throughout the project; Barbara Davis and three anonymous reviewers for insightful comments on earlier versions of the manuscript; and the parents and infants who participated in the study.

Correspondence concerning this article should be addressed to Jana M. Iverson, Department of Psychology, University of Pittsburgh, 3415 Sennott Square, 210 S. Bouquet St., Pittsburgh, PA 15260. Electronic mail may be sent to jiverson@pitt.edu. co-occurring speech, such that the stroke, or active phase, of the gesture is executed just as the related word or phrase is articulated (McNeill, 1992). Gesture and speech, in other words, are closely timed with one another, and this relationship persists even in the face of severe disruptions in the temporal organization of speech. Observations of chronic stutterers, for example, indicate that gesture execution comes to a halt during bouts of stuttered dysfluency; if the hand has begun to rise in anticipation of gesture production, it is held in place until the end of the stuttered bout (Mayberry \& Jaques, 2000).

The temporal relationship between gesture and speech has received considerable attention from researchers (e.g., Kita, 2000; Mayberry \& Jaques, 2000; Nobe, 2000), and a recent study suggests that the adult pattern of gesture-speech synchrony is evident by the time children make the transition to two-word utterances (Butcher \& Goldin-Meadow, 2000). Currently, however, we know little about the infant origins of this phenomenon. The small amount of evidence that can be brought to bear on this issue is indirect and comes from two sources: research on the relationship between vocal and motor activity in early infancy and research on the relationship between production of rhythmic manual movement and the emergence of reduplicated babbling in later infancy (between 6 and 8 months; e.g., Oller, 1980; Stark, 1980).

Connections between the vocal and motor systems appear to be in place early in development. (The use of the terms vocal and motor reflects the fact that the two systems are anatomically distinct. This choice of terminology in no way implies that the systems are separate, as motor activity is clearly involved in the production of speech and vocalization.) The Babkin reflex, for example, can be elicited

(C) 2004 by the Society for Research in Child Development, Inc. All rights reserved. 0009-3920/2004/7504-0006 
in newborns by applying pressure to the palm; infants react to this manual stimulation by opening their mouths (Babkin, 1960). When newborns bring their hands to the facial area to introduce the fingers for sucking, they open the mouth as the hand is moving toward the facial area, in anticipation of its arrival (Butterworth \& Hopkins, 1988; Lew \& Butterworth, 1997). And during face-to-face infantmother interaction, index finger extensions in infants between the ages of 9 and 15 weeks are especially likely to co-occur with vocalization or mouthing movements (Fogel \& Hannan, 1985).

Among older infants, increased production of upper limb rhythmicities has been shown to relate to age of onset of reduplicated babbling (production of repeated sequences of identical syllables (e.g., [bababa]; Cobo-Lewis, Oller, Lynch, \& Levine, 1996; Eilers et al., 1993; Ejiri, 1998). Thus, for example, Locke, Bekken, McMinn-Larson, and Wein (1995) have demonstrated that amount of rhythmic arm shaking in an experimental session is related to babbling experience; rate of shaking was relatively low among prebabblers, increased substantially among infants who had just begun to babble, and then declined (but remained above that for prebabblers) among infants who had been babbling for longer periods. Such findings have been interpreted as suggesting that the coincident changes in rhythmicity in infant vocalization and motor behavior reflect control by a common underlying mechanism (see Eilers et al., 1993; Ramsay, 1984, 1985).

The research reported here is part of a larger effort to determine whether vocal-motor linkages in infancy provide the developmental basis for later speech-gesture coexpression. Some of the questions to be addressed are descriptive, originating in part from what is known about the characteristics of the developed gesture-speech system; other questions are motivated by a model of vocal-motor coordination developed by Iverson and Thelen (1999).

Descriptive issues generally derive from work by McNeill (1992, 2000), who has documented four major characteristics of adult gesture-speech coproduction. First, although gesture and speech often convey complementary aspects of an underlying message, they do so simultaneously, temporally linked within the bounds of a single utterance. Second, when adults gesture while speaking, gestures consist primarily of hand, arm, and finger movements: It is relatively uncommon for mature speakers to produce gestures that involve the legs, feet, or whole body. Third, among right-handed speakers (the majority of all speakers), coexpressive gestures tend to be unimanual and to be produced primarily with the right hand (Kimura, 1973a, 1973b). Finally, gestures and speech have a constant relationship in time, with the manual movements of gesture either slightly anticipating or occurring in synchrony with coexpressive speech.

Descriptive questions suggested by these findings include: To what extent do 6- to 9-month-old infants coordinate vocalization with rhythmic movement, and does frequency of coordination vary with infant age? Do rates of coordination differ for manual and nonmanual behaviors, and do these rates vary with age? To what extent do coordinations involving manual and vocal systems involve one versus both arms and right versus left arms? What is the temporal relationship between vocal and rhythmic motor behaviors produced during bouts of coordination? To the extent that infant vocal-motor coordination is a precursor to the mature gesture-speech system, one might expect the overall rate of vocal-motor coordination to increase with age as children begin to approach the onset of first gestures and words. Because gestures that are part of the adult gesturespeech system consist primarily of hand and arm movements, one might expect age changes in rate of coordination to vary for manual and nonmanual systems, with the rate of manual coordinations becoming greater with age. Because adult gestures are largely unimanual and right-handed, one might expect infant rhythmic manual movements in coordination with vocalization to be more likely to be unimanual and right-handed. And because initiation of adult gestures tends to precede or occur in synchrony with speech, one might expect rhythmic movements either to initiate coordination bouts or to begin simultaneously with coordinated vocalizations.

Other questions to be addressed here are derived as predictions from a theoretical model of the development of vocal-motor coordination articulated by Iverson and Thelen (1999). This model has been developed to provide an integrated account of findings related to vocal-motor linkages in infancy with a view to setting the stage for an increased understanding of the infant origins of gesture-speech timing. A critical factor in the coproduction of speech and gesture is the ability to produce controlled, voluntary movements in the two effector systems (the vocal tract and the manual system) and to coordinate these movements in time and space. Thus, Iverson and Thelen have conceptualized the development of the gesture-speech system within the broader context of the development of motor control, with the emergence of gesture-speech coordination viewed as a specific example of the more general problem of movement coordination. The model seeks 
to provide a principled understanding of how the dynamics of change in the strength and stability of early vocal and motor skills can account for the emergence of the ability to link the vocal and manual modes in a single, coordinated behavior with common communicative intent. The model uses four key concepts: coupled oscillators, entrainment, elicitation threshold, and relative activation strength. Each of these is briefly addressed in turn.

\section{Oscillation and Rhythmicity}

Oscillation and rhythmicity are characteristic behaviors of developing motor systems. Neuromotor systems under immature or impaired voluntary control have the tendency to oscillate naturally. In infants, these oscillations take the form of rhythmically organized, repetitive movements (such as shaking, kicking, rocking, and bouncing) and appear to be closely associated with moments of transition from no voluntary control over a limb or body segment to adaptive, intentional control (Thelen, 1979, 1981a, 1981b).

In the vocal system, the properties of rhythmic limb stereotypies of the sort just described are evident in reduplicated babbling. MacNeilage and Davis (2000; MacNeilage, Davis, Kinney, \& Matyear, 1999; see also Meier, McGarvin, Zakia, \& Willerman, 1997) have argued that the repetitive patterns typical of early reduplicated babbling are the product of mandibular oscillations (where each movement cycle constitutes a syllable) in combination with phonation and limited tongue control. As infants gain control of the tongue and its position during mandibular cycles, they begin to widen the repertoire of syllabic patterns that occur in their vocalizations. Thus, rhythmically organized, repetitive activity is a hallmark of both the infant motor and vocal systems during the first year. Assuming an initial coupling between the two systems, one might expect activity in one system to influence, or entrain, that in the complementary system.

\section{Entrainment}

A second key issue addressed in the Iverson and Thelen (1999) model deals with the property of coupled oscillators to entrain one another. When oscillators are coupled, each tries to draw the other into its characteristic oscillation pattern. Entrainment occurs when one oscillator successfully pulls in the activity of the other, resulting in an ordered patterning of coordinated activity reflected in the temporal characteristics of the entrained system.

In infancy, a coupling of the vocal and manual systems appears to exist from early in development.
This suggests that when there is sufficient activation in one component of the coupled vocal-motor system, that component should pull in and entrain the activity of the complementary system. When an infant is engaged in an intense bout of rhythmic limb activity, for example, the level of activation in the motor system may spill over into the vocal system and entrain its activity, resulting in production of a vocalization. This entrainment is dynamic and flexible, such that activation of one system can have various effects on the other - tight temporal synchrony or more loosely coupled influence. To return to the previous example, depending on the level of activity in the motor system, the co-occurring vocalization may be closely timed with the rhythmic limb movement (e.g., a string of repeated syllables, each articulating with a movement cycle in a bout of rhythmic arm banging) or simply overlapping in time with the movement bout (e.g., a short vocalization occurring during a bout of rhythmic arm banging).

\section{Elicitation Threshold and Relative Activation Strength}

In addition to coupled oscillators and entrainment, the Iverson and Thelen (1999) model makes use of two additional concepts: thresholds for eliciting vocal and motor behaviors, and the relative activation strengths of these behaviors. The threshold for a behavior is presumed to be directly related to its ease of performance. For infants, a good indication of the threshold for a given naturally occurring behavior is how often it is performed. Behaviors with a low threshold for performance are seen frequently and in a variety of task contexts. Behaviors with a high threshold, in contrast, are less frequently produced and are thus presumed to be more effortful. An effect of repeated practice is to lower the threshold for performance, making the behavior available at different times and in multiple and variable contexts.

Activation is defined as the relative strength of the behavior once the threshold is reached and the behavior is performed. Relatively novel, unpracticed behaviors are assumed to have relatively low levels of activation. With new forms of behavior, much of the system's energy is spent reaching threshold. The amount of leftover energy is presumably minimal, and the resulting behavior will therefore be relatively weak.

In contrast, more established, well-practiced behaviors can be said to have relatively higher levels of activation; that is, they are strong, stable skills. A critical assumption here is that the dynamic coupling of two effector systems - limbs and vocal tractrequires relatively high levels of activation in one for 
entrainment to occur. Thus, a given behavior must be relatively strong and stable to pull in and entrain the activity of the complementary system.

The importance of relative activation level and the tendency toward mutual vocal-motor entrainment may be manifested empirically in the relationship between babbling onset and occurrence of coordinated bouts of vocalization with rhythmic manual movement. Infants have a long history of producing rhythmic manual movements before the onset of reduplicated babbling. These movements are stable, well practiced, and should therefore exhibit low threshold and relatively high activation strengthjust the conditions for possible entrainment of the vocal system. With the onset of reduplicated babbling, the possibility of mutual vocal-manual entrainment emerges. Rhythmically organized vocalization can now begin to entrain manual movement just as rhythmic manual movement can entrain vocalization. This development, together with the previously documented increase in rhythmic manual activity around the time of babble onset (e.g., Locke et al., 1995; Thelen, 1979), increases the overall likelihood that rhythmic manual movements and vocalizations, especially rhythmic vocalizations, will occur in coordination.

Taken together, and with the assumption that infant vocal-manual coordination is a precursor to the coproduction of speech and gesture, these considerations lead to several predictions. First, given that entrainment results in a coordinated pattern of activity, vocalizations coordinated with rhythmic movement should themselves be especially likely to exhibit rhythmic organization. Second, if vocalmanual coordination is a precursor to speechgesture synchrony, a higher proportion of speech-like vocalizations should be coordinated with manual than with nonmanual rhythmic movement. And third, given the possibility of mutual vocal-manual entrainment in infants who have begun to babble, one would expect the proportion of rhythmic manual movements coordinated with vocalization to be higher for babblers than for prebabblers, even with age controlled (i.e., among infants at the age of babbling onset).

The present cross-sectional study was designed with two main goals in mind. The first was to describe 6- to 9-month-old infants' production of vocalrhythmic movement coordinations. This age range was chosen because it brackets the previously identified period of increased vocal and manual rhythmicity. The second was to gather preliminary data concerning predictions derived from the Iverson and Thelen (1999) model.

\section{Participants}

Forty-seven infants were recruited through local birth announcements and word of mouth; eligible families were contacted by introductory letter and follow-up phone call. Observations were scheduled within a week of each infant's monthly birth date. All of the infants were from full-term, uncomplicated pregnancies; were developing normally (as assessed by parent reports of attainment of major early developmental milestones); and came from monolingual, English-speaking households. There were fifteen 6-month-olds ( 6 girls, 9 boys; $M=6.08$ months, $S D=.17$ ), eleven 7 -month-olds (5 girls, 6 boys; $M=$ 7.14 months, $S D=.18$ ), ten 8 -month-olds (4 girls, 6 boys; $M=7.97$ months, $S D=.30$ ), and eleven 9-month-olds ( 5 girls, 6 boys; $M=9.0$ months, $S D=$ .23). Twenty-three of the infants were first-borns, 17 were second-borns, and 7 were later-borns. Fortythree infants were Caucasian, 3 were African American, and 1 was Asian American.

Given the potential relevance of babbling status to the phenomena under consideration, we assessed whether the individual infants in this sample had begun to produce reduplicated babble. Thus, infants were identified as babblers if they satisfied either of the following criteria: (a) parental report of reduplicated babble production (elicited by interviewing parents about their infants' production of sounds, describing and providing examples of the characteristics of reduplicated babble, and asking whether the infant ever produced similar vocalizations), or (b) production of at least two instances of reduplicated babble (which included at least two-syllable repetitions) during the observation session. Using these criteria, 42 infants were identified as babblers and 5 (all 6-month-olds) as prebabblers.

Because of the possible relevance of babbling status to vocal-motor coordination and our consequent desire to analyze overall patterns of coordination in a sample homogeneous with respect to babbling status, the five 6-month-old infants classified as prebabblers were excluded from all descriptive analyses. The descriptive findings presented next are thus based on a sample of ten 6-month-olds, eleven 7-month-olds, ten 8-month-olds, and eleven 9-month-olds. However, data from the 5 prebabblers are included in subsequent model-driven analyses in which babbling status was a variable.

\section{Procedure}

Infants and primary caregivers were videotaped at home for approximately $25 \mathrm{~min}$ during a semi- 
structured play session. Observations were scheduled for times when parents thought the infant would be most alert and playful. Informed parental consent was obtained before the start of the observation. The session was divided into two major segments that occurred in fixed order: play with rattles and play with toys.

During the first segment, infants were seated on the caregiver's lap and given a series of three rattles to play with for $3 \mathrm{~min}$ each. This context has been used by other investigators to examine changes in rhythmic manual activity as a function of babbling status and infant age (Locke et al., 1995). The rattles were presented in fixed order and included a standard infant rattle with an internal noisemaker, a set of large plastic keys, and a toy consisting of a set of colorful balls contained within a clear plastic dome mounted on a plastic base.

During play with toys, infants and caregivers engaged in a series of four activities in the following fixed order: book reading, play with a standard toy set provided by the experimenters (a large, colorful rattle and a set of plastic stacking rings), play with the infants' favorite toys, and face-toface interaction during a familiar game or routine. Activities were presented for approximately $5 \mathrm{~min}$ each. For the book-reading segment, infants were seated in the caregiver's lap. All other activities were carried out on the floor. Over the course of the session, infants were naturally observed in a variety of self-adopted postures, including independent sitting, reclining, standing, prone, supine, and on all fours.

\section{Coding}

All infant vocalizations and rhythmic limb movements were coded from the videotapes. Infant posture was also tracked throughout the session. The criteria used in identifying limb movements, vocalizations, and vocal-rhythmic movement coordinations are described next.

Rhythmic limb movements. Rhythmic limb movements were defined as movements repeated in the same form at least three times at regular, short intervals of approximately $1 \mathrm{~s}$ or less (Thelen, 1979). The onset and offset times of each such movement bout were identified and the presence of co-occurring vocalizations noted. Rhythmic movements were further classified on the basis of involved body segment(s) (e.g., both legs, right arm, head, torso) and form. Categories for classifying the form of rhythmic movements were based on Thelen (1979) and are described in the Appendix. Only movement types that were actually observed among the infants in this study are included.

Vocalizations. Coding of vocalizations was completed in two phases. These phases respectively provided the data for the descriptive and modeldriven analyses reported later. In the first phase, coders identified the occurrence of vocalizations by recording onset and offset times for each vocalization. Vocalization boundaries were determined by the occurrence of an audible breath, or if no breath occurred, by a silence lasting $1 \mathrm{~s}$ or longer (Lynch, Oller, Steffens, Levine, et al., 1995). Consistent with standard practice (e.g., see Nathani \& Oller, 2001), fixed signals (e.g., laughter, crying) and vegetative sounds (e.g., coughing, burps) were excluded from these data. As subsequently employed in this manuscript, therefore, the term vocalization refers to prespeech vocal utterances excluding fixed signals and vegetative noises. This is generally equivalent to the term protophone as employed by Oller (2000) and others. Vocalization so defined served as the unit of analysis for the descriptive results presented later.

In Phase 2, all vocalizations produced during the first $20 \mathrm{~min}$ of the observation were transcribed independently for a randomly chosen subset of infants ( $n=40,10$ at each age) by a trained coder using broad phonetic transcription and International Phonetic Alphabet (IPA) notation. This yielded a set of prespeech vocalizations that contained a wide variety of precanonical and canonical infant sounds, including vowels (both quasi-resonant and fully resonant), single syllables, and repeated syllables (Fagan \& Iverson, 2003; see the Appendix).

From among this broad set of vocalizations, those utterances containing at least two repeated syllables were identified, labeled as consonant-vowel (CV) repetitions, and employed as the unit of analysis in evaluating model-driven predictions. Because we had no a priori reason to believe that model-driven predictions might vary as a function of repetition type, no distinction was made within the category of $\mathrm{CV}$ repetitions between utterances containing repetitions of a single syllable (i.e., reduplicated babble) and those containing syllable repetition with sound variation (i.e., variegated babble). Marginal babble (i.e., production of primitive syllables with slow formant transitions generally "not produced repetitively in sequences of syllables"; Oller, 2000, p. 176) was effectively excluded from these data by virtue of the fact that we restricted analysis to utterances containing at least two repetitions. In effect, then, our category of $\mathrm{CV}$ repetitions corresponds to a subset of vocalizations that have typically been termed $c a$ nonical babble in previous work (e.g., Oller, 1980, 
2000). Oller's (1980, 2000) definition of canonical babble rests primarily on the issue of well formedness; in his classification system, utterances consisting of single syllables may be considered canonical babble if the syllable is well formed. CV repetition as employed in this paper therefore refers to a subset of utterances that might be classified as canonical babble.

Vocalization-rhythmic movement coordinations. Vocalizations and rhythmic movements were considered to be coordinated when the behaviors shared some degree of temporal overlap. When coding infant vocal and rhythmic motor behaviors in a given session, coders recorded instances of individual vocal and motor behaviors (i.e., those that were produced in isolation) as well as vocal and motor behaviors that co-occurred in time with other target behaviors. When vocal and motor behaviors $\mathrm{CO}^{-}$ occurred, they did so in one of two ways: (a) the onset of one behavior (e.g., a vocalization) occurred during an ongoing bout of another behavior (e.g. rhythmic kicking), or (b) the onsets were simultaneous. Although an instance of coordination by definition involved at least two behaviors (a vocalization and a rhythmic movement), there were a few instances of coordinations involving multiple vocal and motor behaviors. The frequency of such coordinations was so low, however, that they were not separately analyzed.

Reliability. To assess intercoder reliability, a second trained coder independently coded $10 \%$ of the videotaped data. Mean percentage agreement was $88 \%(N=324)$ for identifying the occurrence of a rhythmic movement, $90 \%(N=663)$ for identifying the occurrence of a vocalization, $86 \%(N=234)$ for classifying rhythmic movement types, $79 \%$ for classifying vocalization types $(N=512)$, and $95 \%(N=$ 85) for identifying co-occurrence between rhythmic movements and vocalizations.

\section{Results}

\section{Infant Vocal-Motor Coordination: Descriptive Analyses}

We begin by presenting data relevant to the four descriptive questions derived from the literature on the adult gesture-speech system. These questions have to do with frequency of vocal-motor coordination, relative rates of coordination involving manual versus nonmanual behaviors, laterality of manual behaviors coordinated with vocalization, and the timing relationship between coordinated vocal and motor behaviors. If infant vocal-motor coordination shares features of the adult system, the hypothesis that vocal-motor coordination serves as a developmental precursor to mature gesturespeech coproduction is strengthened.

Frequency of vocal-motor coordination. Forty-one of the 42 infants in this study coordinated vocalizations with rhythmic limb activity, and they did so frequently. The single infant (a 7-month-old) who did not produce any coordination bouts was excluded from the analyses. For purposes of the present report, data are presented collapsed across movement types. Rhythmic limb movements were broadly classified into two major categories: manual (including arm and hand movements) and nonmanual (including leg, foot, torso, and head movements).

Inspection of the proportions of rhythmic movements and vocalizations in coordination (i.e., the number of rhythmic motor/vocal behaviors in coordination divided by the total number of rhythmic motor/vocal behaviors) indicated that approximately $20 \%$ of all rhythmic movements were coordinated with vocalization, a tendency that remained relatively stable with infant age $\left(M_{6}=.18, S D=.11\right.$; $M_{7}=.23, S D=.20 ; M_{8}=.25, S D=.25 ; M_{9}=.21, S D=$ .17). With regard to vocalizations, there was a tendency for the proportion of coordinated vocalizations to increase with infant age, but the trend was not statistically reliable $\left(M_{6}=.07, S D=.05 ; M_{7}=.11\right.$, $\left.S D=.08 ; M_{8}=.14, S D=.13 ; M_{9}=.14, S D=.14\right)$.

Rate of coordination of manual versus nonmanual behaviors. Production of vocal-rhythmic manual and vocal-rhythmic nonmanual coordinations was first examined by calculating the rate of occurrence of coordinated movements separately for each movement category (i.e., the total number of coordinated movements produced, divided by duration of the observational segment). Rates were calculated separately for the rattles and play contexts. These data are presented in Figure 1.

Consistent with expectation, rate of coordination was generally higher for manual than for nonmanual movements for all age groups. Rate of coordination was also generally higher for rattles than for play, and in the rattles condition, for older than for younger infants. A 4 (age) $\times 2$ (body segment) $\times 2$ (context) repeated-measures analysis of variance (ANOVA) carried out on these data revealed reliable main effects of age, $F(3,37)=12.05, p<.01$; body segment, $F(3,37)=32.38, p<.01$; and context, $F(3$, $37)=84.69, p<.01$. The Age $\times$ Context and Age $\times$ Body Segment interactions were also significant, $F(3$, $37)=17.30, p<.01$, and $F(3,37)=4.20, p<.02$, respectively. Simple effects analyses carried out to assess the source of the Age $\times$ Context interaction indicated that in the rattles context, 8- and 9-montholds coordinated rhythmic movements with vocali- 


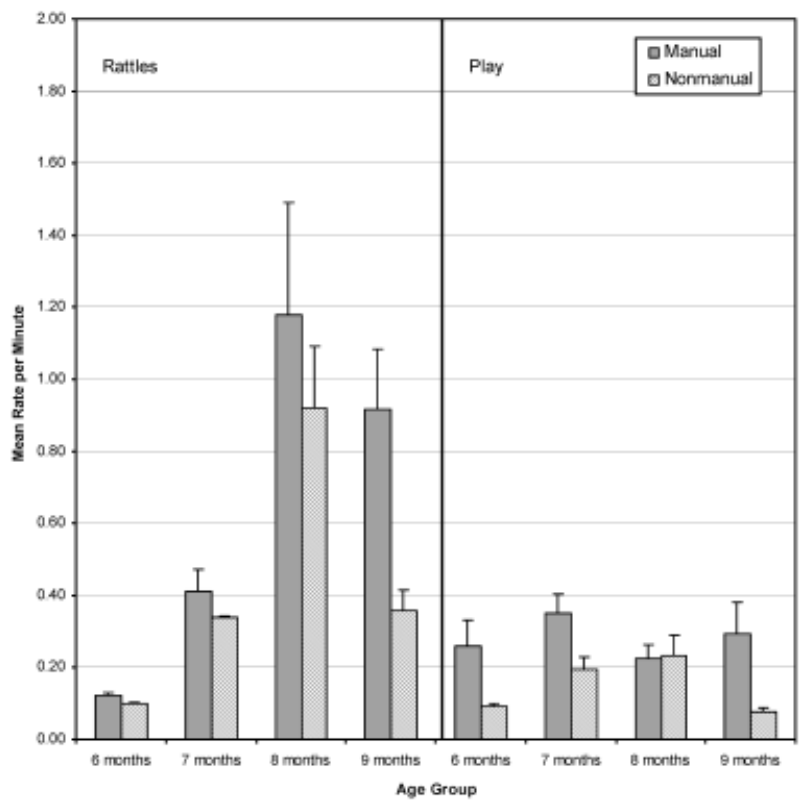

Figure 1. Rate of vocal-rhythmic manual and vocal-rhythmic nonmanual coordination.

zation at significantly higher rates than did 6- and 7 -month-olds $\left(M_{6 / 7}=.27, S D=.18 ; M_{8 / 9}=.81, S D=.56\right.$, $p<.001)$. Neither 8- and 9-month-olds $\left(M_{8}=.67\right.$, $\left.S D=.53 ; M_{9}=.94, S D=.58, n s\right)$ nor 6 - and 7-montholds $\left(M_{6}=.15, S D=.07 ; M_{7}=.39, S D=.18, n s\right)$ differed respectively from one another. Rates of coordination did not differ reliably among the four age groups in the play context $\left(M_{6}=.28, S D=.20 ; M_{7}=\right.$ $.37, S D=.27 ; M_{8}=.26, S D=.11 ; M_{9}=.43, S D=.37$ ).

One possible explanation for the higher rate of coordination observed in the rattles context that would not involve entrainment of vocalization by rhythmic motor activity is that in rattle play, rhythmic movement generally results in contingent and rhythmic sound, and that vocalization (and therefore the likelihood of coordination) may be enhanced by this contingency. One way to assess this would be to compare the proportions of rhythmic movements coordinated with vocalization in the rattles context with contingent rhythmic sound with that in the play context, where no such contingency exists. To this end, mean proportions of rhythmic movements coordinated with vocalization were calculated separately for infants in each age group for the two contexts and compared. These means did not differ significantly by age or by context (for rattles: $M_{6}=$ $.24, S D=.22 ; M_{7}=.17, S D=.23 ; M_{8}=.22, S D=.28$; $M_{9}=.17, S D=.24 ;$ for play: $M_{6}=.18, S D=.11$; $M_{7}=.23, S D=.20 ; M_{8}=.25, S D=.25 ; M_{9}=.21, S D=$ .17), suggesting that the higher rate of coordination in rattles cannot be simply explained by the presence of a rhythmic auditory consequence to rhythmic movement.

With regard to the Age $\times$ Body Segment interaction, simple effects analyses revealed that 8-montholds coordinated nonmanual movements with vocalization at a significantly higher rate than did the 6-, 7-, and 9-month-olds combined $\left(M_{8}=.21\right.$, $\left.S D=.10 ; M_{6 / 7 / 9}=.10, S D=.17, p<.008\right)$ but did not differ from the combined group in rate of rhythmic manual coordination $\left(M_{8}=.28, S D=.14 ; M_{6 / 7 / 9}=.27\right.$, $S D=.38$ ). Six-month-olds produced a significantly lower rate of rhythmic nonmanual movements in coordination than did 7- and 9-month-olds combined $\left(M_{6}=.02, S D=.01 ; \quad M_{7 / 9}=.13, S D=.23\right.$, $p<.05)$, and the difference in rate of rhythmic manual coordination tended toward significance $\left(M_{6}=.16, S D=.06 ; M_{7 / 9}=.30, S D=.45, p<.09\right)$. Seven- and 9-month-olds did not differ from one another either in rate of coordination of rhythmic manual $\left(M_{7}=.30, S D=.16 ; M_{9}=.33, S D=.13\right)$ or rhythmic nonmanual movements $\left(M_{7}=.12, S D=.05\right.$; $M_{9}=.14, S D=.07$ ).

Note that differences in production of rhythmic manual versus nonmanual movements were not simply a function of infants adopting postures that permit rhythmic arm activity but constrain rhythmic movements of the legs, torso, and head. Examination of the relative proportions of rhythmic movements produced by different body segments in sitting and nonsitting (prone, supine, standing, etc.) postures (data summed across age) revealed that these distributions were generally parallel (sitting: $M_{\text {arms }}=$ $.87, M_{\text {legs }}=.03, M_{\text {torso }}=.06, M_{\text {head }}=.04$; nonsitting: $\left.M_{\text {arms }}=.68, M_{\text {legs }}=.17, M_{\text {torso }}=.11, M_{\text {head }}=.04\right)$. Although leg and torso movements were more common and manual movements were less common in nonsitting than in sitting postures, the relative preponderance of manual rhythmicity was clearly not simply a function of manual facilitation in the sitting position.

The next analysis examined whether the observed differences in rate of vocal-rhythmic movement coordination with infant age could be accounted for by systematic differences in length of coordination bout as a function of age. Thus, for example, rate of coordination bouts might have been lower for younger infants in the rattles context because younger infants produced more temporally extended coordination bouts (i.e., for a given session length, opportunity to produce separate coordinations is reduced in direct proportion to average duration of coordination).

To assess this possibility, average lengths of coordination bouts were calculated separately for each infant for the rattles and play contexts. This was done by dividing the total number of vocal-rhythmic 
movement coordination bouts (collapsed across body segment) by the sum of the durations of the bouts (in seconds). In general, coordination bout durations were similar across all age groups and in both contexts (for rattles: $M_{6}=1.93, S D=1.61 ; M_{7}=$ $1.58, S D=.45 ; M_{8}=1.84, S D=.98 ; M_{9}=1.57, S D=$ .62 ; for play: $M_{6}=1.17, S D=.47 ; M_{7}=1.23, S D=.53$; $M_{8}=2.16, S D=1.22 ; M_{9}=1.86, S D=1.35$ ). A 4 (age) $\times 2$ (context) repeated-measures ANOVA revealed no reliable age or context effects; the interaction was also not significant. Thus, the observed age differences in rate of coordination are not simply a reflection of age-related variation in coordination bout length.

Laterality. The data presented thus far indicate that, consistent with features of the mature gesturespeech system, 6- to 9-month-old infants frequently coordinate vocalization with motor activity, with a majority of these coordination bouts involving rhythmic manual activity. We next examined whether the patterns of laterality in infant vocalrhythmic manual coordinations mirrored those observed among adult speakers (i.e., most coexpressive gestures are unimanual and executed with the right hand; Kimura, 1973a, 1973b). Because inspection of group means revealed no differences in distributions of unimanual versus bimanual and right versus left arms by context, data for these analyses are reported collapsed across contexts.

Results revealed first that for infants at all ages, a greater proportion of coordinated rhythmic manual movements involved one arm rather than both arms. It is interesting that this tendency was stronger among 6-month-olds $\left(M_{6}=.85, S D=.32\right)$ than among infants at other ages $\left(M_{7}=.62, S D=.34 ; M_{8}=.62\right.$, $\left.S D=.44 ; M_{9}=.64, S D=.36\right)$. A one-way ANOVA confirmed this age difference, $F(3,34)=3.09, p<.05$. Post hoc Tukey tests indicated that source of the effect was in the significantly higher proportion of single-arm movements produced by 6-month-olds relative to 9 -month-olds $(p<.04)$; no other comparisons were reliable. This pattern generally mirrored that observed among rhythmic manual movements that were not coordinated with vocalization (single arm: $M_{6}=.82, S D=.18 ; M_{7}=.81, S D=.23 ; M_{8}=.72$, $S D=.24 ; M_{9}=.66, S D=.26$ ).

With regard to laterality, the majority of infants' single-arm coordinations involved the right arm $\left(M_{6}=.54, S D=.43 ; M_{7}=.60, S D=.37 ; M_{8}=.72\right.$, $S D=.11 ; M_{9}=.61, S D=.33$ ). Age differences were not statistically reliable; thus, data were collapsed across age groups to assess the significance of the proportion of right- versus left-arm movements. Results indicated that when only one arm was involved in coordination, infants were significantly more likely to coordinate vocalizations with rightarm movements $(M=.62, S D=.32)$ than with leftarm movements $(M=.38, S D=.32), t(41)=3.39$, $p<.003$. In addition, among infants who produced at least one right-arm movement, the proportion of right-arm movements was significantly higher for coordinated rhythmic manual movements $(M=.62$, $S D=.32)$ than for rhythmic manual movements alone $(M=.58, S D=.32), F(3,34)=4.35, p<.05$.

Timing. When adults produce gestures when speaking, those gestures either anticipate or occur in synchrony with coexpressive speech. Our final descriptively motivated analysis examined whether this temporal patterning was evident in infants' coordination bouts. All coordination bouts were classified according to whether they were movement initiated (e.g., infant bangs arm, then begins to vocalize), vocalization initiated (e.g., infant vocalizes, then begins to kick), or synchronous (e.g., infant begins to swing arms and vocalize at the same time), and the relative proportions of each bout type were calculated. In the absence of age differences, data were collapsed across groups. Consistent with our hypothesis and with patterns observed in adult gesture-speech coordinations, the vast majority of infants' vocal-motor coordination bouts were either movement initiated or synchronous $(M=.83$, $S D=.20)$.

\section{Infant Vocal-Motor Coordination: Model-Driven Predictions}

The second major goal of the study was to test predictions derived from the model of infant vocalmotor interaction proposed by Iverson and Thelen (1999). These predictions had to do with effects of entrainment on vocalization properties, differences in the types of vocalizations coordinated with manual versus nonmanual behaviors, and the relationship between reduplicated babble onset and vocalrhythmic manual coordinations. Data relevant to these hypotheses are presented next.

Entrainment and vocalization properties. As previously discussed, a characteristic of coupled oscillators is that each tries to draw the other into its characteristic pattern of activity. When entrainment occurs, activity patterns in the entrained system may assume properties of the entraining system. If entrainment is the mechanism underlying coordination of vocalization with rhythmic motor activity, vocalizations coordinated with rhythmic movement should be more likely to be rhythmically organized than vocalizations without accompanying rhythmic movement. 
Infant vocalizations exhibit rhythmic organization from very young ages, and this rhythmicity is apparent both within utterances (i.e., syllables organized into utterances) and across utterances (i.e., utterances organized into prelinguistic phrases; Lynch, Oller, Steffens, \& Buder, 1995). However, because our hypothesis had to do with vocal-motor entrainment, specifically whether individual vocalizations co-occurring with rhythmic motor activity are especially likely to exhibit rhythmic organization, we focused on vocalizations containing CV repetition. We did so because the defining characteristic of $\mathrm{CV}$ repetition is rhythmicity at the within-utterance level (e.g., MacNeilage \& Davis, 1993; Oller, 2000). In addition, $\mathrm{CV}$ repetitions share some of the features of the rhythmic motor behaviors under consideration: They are a product of rhythmic jaw movement (e.g., Davis \& MacNeilage, 1995; MacNeilage \& Davis, 1993), and within bouts of CV repetitions, the rate of repetition is similar to that of rhythmic motor behaviors (Oller, 2000, p. 114). Inspection of the data revealed that, on average, the proportion of vocalizations containing CV repetitions accounted for $2 \%$ to $19 \%$ of all infant vocalizations.

To examine whether vocalizations co-occurring with rhythmic activity were more likely to exhibit rhythmic organization than vocalizations without accompanying rhythmic movement, we contrasted the relative frequencies of vocalizations containing $\mathrm{CV}$ repetitions with and without accompanying rhythmic movement. Inspection of the data indicated that the effects of accompanying rhythmic movement did not differ as a function of age (i.e., there was no Age $\times$ Movement Status interaction); thus, data were collapsed across age groups. Results of this analysis revealed that among the 15 infants who had at least one vocalization containing $\mathrm{CV}$ repetition in each category (i.e., with and without rhythmic movement), CV repetitions were significantly more likely to occur with rhythmic movement $(M=.24, S D=.13)$ than without rhythmic activity $(M=.17, S D=.14), t(14)=-1.85, p<.05$, one-tailed.

Vocalizations coordinated with rhythmic manual versus rhythmic nonmanual behaviors. The next prediction had to do with the specificity of the relationship between rhythmic manual movements and production of $\mathrm{CV}$ repetitions. For this analysis, we again focused on vocalizations containing $\mathrm{CV}$ repetitions inasmuch as previous research has indicated that these are the most speech-like of infant vocalizations (e.g., Oller, 2000) and that they are reliable predictors of future language development (e.g., Stoel-Gammon, 1998). If infant vocal-manual coordination is a precursor to mature gesture-speech coproduction, and if $\mathrm{CV}$ repetition is a precursor to speech, then among $\mathrm{CV}$ repetitions coordinated with rhythmic movement we would expect to find a higher proportion coordinated with rhythmic manual than with rhythmic nonmanual movement.

Findings were consistent with this prediction. With data collapsed across age groups (infants who produced no $\mathrm{CV}$ repetitions with rhythmic movement were excluded from this analysis), the proportion of $\mathrm{CV}$ repetitions coordinated with rhythmic manual movements was significantly greater than that coordinated with rhythmic nonmanual movements $\left(M_{\text {manual }}=.58, S D=.49 ; \quad M_{\text {nonmanual }}=.42\right.$, $S D=.49), t(14)=1.86, p<.05$, one-tailed.

The relationship between babbling onset and vocalmanual coordination. Our final analysis focused on changes in vocal-manual coordination in relation to the emergence of reduplicated babble. According to the model, mutual entrainment of the vocal and manual systems becomes possible with the onset of reduplicated babble. With both systems able to extend their influence to the complementary system, the likelihood of coordination between vocalization and manual behaviors should therefore be higher in babblers relative to prebabblers of the same chronological age. To test this prediction, we compared data from the ten 6-month-olds previously identified as babblers with those from the five 6-month-old prebabblers who were excluded from the previously reported descriptive analyses. A Mann-Whitney $U$ test indicated that the two groups of 6-month-olds did not differ significantly in age, $U=17$, ns. We then calculated the proportion of rhythmic manual movements coordinated with vocalization for each infant (i.e., the number of rhythmic manual movements in coordination divided by the total number of rhythmic manual movements) and compared babblers with prebabblers.

Consistent with our prediction, the median proportion of rhythmic manual movements coordinated with vocalization for babblers $(M d n=.18, S D=.08)$ was slightly more than twice as high as that for prebabblers $(M d n=.08, S D=.09) ; 7$ of the $10 \mathrm{bab}$ blers fell above the median for prebabblers, whereas only 1 prebabbler fell above the median for babblers, Fisher's exact test $p=.05$, one-tailed. Evaluation of this comparison with babbling status defined as both parental report of babble and production of at least two babbled vocalizations during the observation (babblers: $n=7$; prebabblers: $n=8$ ) revealed a similar, statistically reliable pattern, Fisher's exact test $p=.04$, one-tailed. 


\section{Discussion}

This research was designed to examine the interplay between vocal and rhythmic limb activity in 6- to 9month-old infants. The study had two major goals. The first was to address a series of descriptive questions derived from the literature on the adult gesture-speech system by providing a general picture of vocal-motor coordination and its characteristics in relation to infant age, limb system (manual vs. nonmanual), and context. The second was to gather data regarding three hypotheses central to a theoretical model of the development of the gesture-speech system. These predictions had to do with effects of entrainment on vocalization properties, differences in the types of vocalizations coordinated with manual vs. nonmanual behaviors, and the relationship between reduplicated babble onset and vocal-rhythmic manual coordination.

\section{Vocal-Rhythmic Movement Coordination in Infancy}

The results presented here indicate that vocalrhythmic movement coordinations are a robust feature of infant behavior. Bouts of vocal-rhythmic movement coordination occurred frequently and were produced by all but 1 of the infants in this study. Coordination was observed in both rattles and play contexts, regardless of whether infants were playing with sounding or nonsounding objects.

Only one other study has attempted to document vocal-motor coordination in infants. In a longitudinal study, Ejiri and Masataka (2001) examined cooccurrences between vocalization and motor activity in 4 infants observed monthly in naturalistic in-home play sessions between the ages of 6 and 11 months. They coded vocalizations and four types of motor activity (mouthing, manipulating, banging, and rhythmic actions) and noted all instances of co-occurrence between vocal and motor behaviors, defined as a temporal overlap between behaviors of more than $1 \mathrm{~s}$. The authors reported that, in general, a higher percentage of vocalizations co-occurred with rhythmic movements than with other types of motor behaviors. In addition, there was a peak in the percentage of vocalizations co-occurring with rhythmic movement that coincided with reduplicated babble onset.

Data from this study are generally consistent with these findings. There are, however, two ways in which the results of the current research extend those of Ejiri and Masataka (2001) and one important way in which they differ. First, coordination results presented by Ejiri and Masataka are described in terms of the percentage of vocalizations that co-occurred with motor behavior. The present study additionally provides data on the frequency with which bouts of vocal-motor coordination occur in spontaneous infant behavior. These data indicate that by 7 months of age, vocal-motor coordination is a stable component of infants' behavioral repertoires, occurring regularly in contexts that vary widely in the extent to which they may encourage production of rhythmic behaviors. Second, rhythmic actions are described by Ejiri and Masataka without regard to either body segment or context. The present study provides data on the extent to which rhythmic movement/vocalization co-occurrences differ as a function of both limb and context.

The primary difference between results reported in this study and those of Ejiri and Masataka (2001) involves their report that from ages 6 to 11 months, percentage of vocalizations co-occurring with rhythmic movement decreased with age. By contrast, results presented here indicate a tendency (albeit nonsignificant) toward an age-related increase in the percentage of vocalizations in coordination with rhythmic movement. The source of these differences may lie in methodological variations between the studies, specifically in the criteria used to identify instances of vocal-motor co-occurrence in the two studies. Ejiri and Masataka defined instances of cooccurrence as those in which vocalization overlapped with motor activity for at least $1 \mathrm{~s}$. In the present study, however, any instance of overlap between vocal and motor behaviors was counted as a co-occurrence, regardless of its duration. If, as older infants approach the transition to first words, vocalizations become progressively shorter, approximating more punctate, word-like forms, the number of vocalizations of sufficient duration to meet the Ejiri and Masataka criterion for coordination would decline relative to the total number of vocalizations. The decrease in percentage of vocalization in cooccurrence observed by Ejiri and Masataka (but not in the present study) may, in other words, be a joint function of their coding criterion and of developmental change in the duration of infant vocalizations rather than a true decline in vocal-motor coordination.

If early vocal-motor coordinations are a developmental precursor to the coordinated gesturespeech pattern, as infants approach the age of first gesture and word production, one might expect to find an increase in coordination of rhythmic manual movements and a corresponding decrease in the frequency with which rhythmic nonmanual movements are coordinated. Some support for this prediction comes from the fact that although rate of 
coordination for rhythmic manual movements tended to increase in a relatively linear fashion with age, the shape of age-related change in coordination of rhythmic nonmanual movement was generally curvilinear, with the highest frequency evident among 8-month-olds, followed by a decrease in 9-month-olds. This trend was evident in both rattle and play contexts.

Evidence for a potential developmental relationship between early vocal-motor and later gesturespeech coordinations comes from three additional observations. First, at all ages, infants were more likely to coordinate single-arm than both-arm rhythmic movements with vocalization. Second, infants' patterns of laterality in coordinated manual movements were not random: At all four ages, the proportion of right-arm movements in coordination was reliably greater than that of left-arm movements. This preference for coordinating vocalization with right-arm movements is parallel to patterns observed in the gesture-speech coproductions of adults: In right-handed speakers, most gestures are executed with a single arm, and most often with the right arm (Kimura, 1973a, 1973b). Third, the vast majority of infant coordinations displayed the pattern of temporal organization evident in adult gesture-speech coproductions, with motor activity either slightly anticipating or synchronous with vocalization onset. Although this evidence is only circumstantial, it is suggestive. Clearly, an important goal for future work will be to examine the extent to which patterns of infant vocal-motor coordination may be related to the emergence and development of the gesture-speech system. A longitudinal study designed to address this issue is currently under way.

\section{Entrainment and Vocal-Motor Coordination: Theoretical Predictions}

A second goal of this study was to gather preliminary data concerning specific predictions derived from a theoretical model of the infant vocal and motor systems as coupled oscillators that can mutually entrain one another. According to the model, entrainment is the mechanism of developmental change in the strength and specificity of vocalmotor coordination. However, entrainment and the likelihood with which it will occur are directly influenced by the developmental history of behaviors in the component systems. Thus, the extent to which one system can entrain activity in the complementary system is influenced by the relative strength and stability of behaviors in the component systems.
There were three findings relevant to predictions derived from the model.

The first finding was that relative to vocalizations without co-occurring rhythmic movement, a higher proportion of vocalizations with rhythmic movement consisted of $\mathrm{CV}$ repetitions. This result can be interpreted as evidence for the entrainment of the infant vocal and motor systems as coupled oscillators. When infants engage in bouts of rhythmic limb activity, the rhythmic quality of these movements influences vocal activity, such that co-occurring vocalizations are especially likely to exhibit rhythmic organization. Further empirical support for this interpretation comes from acoustic analyses comparing infant vocalizations that co-occurred with rhythmic motor activity with vocalizations produced without accompanying rhythmic movement (Ejiri \& Masataka, 2001). Relative to vocalizations produced alone, vocalizations co-occurring with rhythmic movement had significantly shorter syllable lengths and formant-frequency transitions. Rapid, wellformed formant transitions between consonant like and vowel-like sounds is one of the dimensions that distinguish the syllabic structure of canonical babble (and mature syllables in general) from precanonical vocalizations (Oller, 2000). This finding provides direct evidence for the influence of rhythmically organized limb activity on characteristics of infant vocalizations.

The second finding was that a majority of the CV repetitions that co-occurred with rhythmic movement occurred in coordination with rhythmic manual movement. This is consistent with the possibility that mature gesture-speech coproduction emerges from infant vocal-motor (especially vocal-manual) coordination. Production of CV syllables is considered predictive of later language development (e.g., see Stoel-Gammon, 1992, 1998,for reviews), and CV repetitions, which contain $\mathrm{CV}$ syllables produced with adult-like timing, share properties of mature speech (e.g., Ejiri \& Masataka, 2001; Stoel-Gammon, 1998). Our data suggest a link between more speechlike vocalizations and manual activity that may be a precursor to coordinated manual movement of the sort involved in adult gestures. Although further work is needed to determine whether the relationship between CV repetition and manual activity continues to strengthen as infants approach the time of emergence of first gestures and words (a pattern that would be expected if these early vocal-manual coordinations are in fact precursors to the speechgesture system), our data provide evidence of differences in vocalizations that co-occur with manual versus nonmanual activity. 
The third result had to do with changes in vocalmanual coordination in relation to onset of reduplicated babble. We found that 6-month-old prebabblers coordinated vocalizations with rhythmic manual activity at a significantly lower rate than did 6-month-old babblers. This is consistent with the model-driven claim that onset of reduplicated babbling provides opportunity for mutual entrainment of the vocal and manual systems, thereby resulting in an increase in the overall likelihood of entrainment and a strengthening of the tendency to coordinate vocalizations with manual behaviors.

The principal results of this study support the following developmental scenario. Infants have a long history of producing rhythmic manual behaviors before babbling onset; they are sufficiently strong and well practiced to activate and entrain vocal activity. During bouts of entrainment, the rhythmically organized nature of these upper limb behaviors may become echoed in vocalization, such that vocalizations begin to take on the rhythmically organized quality that is characteristic of reduplicated babble (e.g., Ejiri \& Masataka, 2001). Once infants begin to produce reduplicated babble, bouts of vocal-manual coordination become increasingly frequent, and the vocal-manual link is progressively strengthened. A few months later, the tendency to coordinate vocalization with rhythmic nonmanual behaviors begins to decline. Thus, by the time infants reach the ages of 9 to 12 months, the time of first gesture and first word onset, the link between vocalization and manual activity is strong, specific, and stable, and is available to be used for communication.

Although longitudinal work is needed to define the nature of the relationship between early vocalmotor and later speech-gesture coordinations in individual infants, the results reported here provide preliminary evidence that the vocal and motor systems (in particular, the manual system) are linked and mutually influential from early in infancy. They also underscore the fruitfulness of an integrated approach to the study of early vocal and motor development, two research areas that have been largely independent. Such an approach will increase our understanding of the processes underlying the $\mathrm{CO}^{-}$ production not only of speech and gesture but of coordinated infant movements in general.

\section{Appendix}

\section{Coding Categories for Rhythmic Limb Movements and Vocalizations}

Rhythmic limb movements

\begin{tabular}{|c|c|c|}
\hline Limb group & Movement & Description \\
\hline \multirow[t]{3}{*}{ Legs and feet } & Both legs kick & Flexion and extension of the two legs, either simultaneously or in alternation. \\
\hline & Single leg kick & Flexion and extension of one leg. \\
\hline & Foot rub & The medial surface of the heel and foot rub against the ankle and foot of the opposite leg. \\
\hline \multirow[t]{2}{*}{ Torso } & Bounce & Torso moves up and down. \\
\hline & Rock & Back-and forth movement. \\
\hline \multirow[t]{3}{*}{ Arms } & Swing & $\begin{array}{l}\text { Vertical movement of the arm from the shoulder with no object in the } \\
\text { hand and no contact with an object or surface. }\end{array}$ \\
\hline & Shake & $\begin{array}{l}\text { Identical to swing, except with an object held in the hand. No contact } \\
\text { with an object or surface. }\end{array}$ \\
\hline & Bang & $\begin{array}{l}\text { Same general movement as swing, but the hand or an object held in the hand makes firm } \\
\text { contact with another object or a surface on the downward stroke. }\end{array}$ \\
\hline \multirow[t]{2}{*}{ Hands } & Flex & Bending and extending of the wrist. \\
\hline & Twist & Rotation of the wrist back and forth. \\
\hline \multirow[t]{2}{*}{ Head } & Roll side to side & Lateral rotation of head (similar to headshake). \\
\hline & Roll front to back & Forward-backward movement (similar to head nod). \\
\hline \multicolumn{3}{|l|}{ Vocalizations } \\
\hline \multicolumn{2}{|l|}{ Category } & Example \\
\hline \multicolumn{2}{|c|}{ Fixed signals ${ }^{\mathrm{a}}$} & Laughter, crying \\
\hline \multicolumn{2}{|c|}{$\begin{array}{l}\text { Fixed signals }{ }^{\mathrm{a}} \\
\text { Vegetative sounds }^{\mathrm{a}}\end{array}$} & Sneezing, burping, hiccups \\
\hline \multicolumn{2}{|c|}{ Single sounds } & {$[\&],[E]$} \\
\hline \multicolumn{2}{|c|}{ Single syllable (one CV unit) } & {$[b V],[u b],[b E m],[\& d \&]$} \\
\hline \multicolumn{2}{|c|}{ Repeated syllables (multiple CV units) } & [d\&d\&], [hEhE], [b\&bV], [d\&b\&], [d\&mV] \\
\hline
\end{tabular}

Note. $\mathrm{CV}=$ consonant - vowel.

${ }^{a}$ These categories were excluded from all data analyses. 


\section{References}

Babkin, P. S. (1960). On the question of the establishment of reflex activity in early postnatal ontogenesis in man. In Central Nervous System and Behavior. Bethesda, MD: National Institutes of Health. [Translated from $\mathrm{K}$ voprosu o stanovlenii reflektornoi deiatel'nosti $\mathrm{v}$ rannem postnatal'nomontogeneze cheloveka, Fiziologicheskii Zhurnal SSSR, 44, 922-927]

Butcher, C. M., \& Goldin-Meadow, S. (2000). Gesture and the transition from one- to two-word speech: When hand and mouth come together. In D. McNeill (Ed.), Language and gesture (pp. 235-257). Cambridge, England: Cambridge University Press.

Butterworth, G., \& Hopkins, B. (1988). Hand-mouth coordination in the new-born baby. British Journal of Developmental Psychology, 6, 303-313.

Cobo-Lewis, A. B., Oller, D. K., Lynch, M. P., \& Levine, S. L. (1996). Relations of motor and vocal milestones in typically developing infants and infants with Down Syndrome. American Journal on Mental Retardation, 100, $456-467$.

Davis, B. L., \& MacNeilage, P. F. (1995). The articulatory basis of babbling. Journal of Speech, Language, and Hearing Research, 38, 1199-1211.

Eilers, R., Oller, D. K., Levine, S., Basinger, D., Lynch, M. P., \& Urbano, R. (1993). The role of prematurity and socioeconomic status in the onset of canonical babbling in infants. Infant Behavior and Development, 16, 297-315.

Ejiri, K. (1998). Relationship between rhythmic behavior and canonical babbling in infant vocal development. Phonetica, 55, 226-237.

Ejiri, K., \& Masataka, N. (2001). Co-occurrence of preverbal vocal behavior and motor action in early infancy. Developmental Science, 4, 40-48.

Fagan, M. K., \& Iverson, J. M. (2003, April). Characteristics of vocalization with and without co-occurring motor activity. Poster session presented at the biennial meetings of the Society for Research in Child Development, Tampa, FL.

Fogel, A., \& Hannan, T. E. (1985). Manual actions of nine to fifteen-week-old human infants during face-to-face interactions with their mothers. Child Development, 56, $1271-1279$.

Iverson, J. M. (1999). How to get to the cafeteria: Gesture and speech in blind and sighted children's spatial descriptions. Developmental Psychology, 35, 1132-1142.

Iverson, J. M., \& Goldin-Meadow, S. (1997). What's communication got to do with it? Gesture in congenitally blind children. Developmental Psychology, 33, 453-467.

Iverson, J. M., \& Goldin-Meadow, S. (1998). Why people gesture when they speak. Nature, 396, 228.

Iverson, J. M., \& Goldin-Meadow, S. (2001). The resilience of gesture in talk: Gesture in blind speakers and listeners. Developmental Science, 4, 416-422.

Iverson, J. M., \& Thelen, E. (1999). Hand, mouth, and brain: The dynamic emergence of speech and gesture. Journal of Consciousness Studies, 6, 19-40.
Kimura, D. (1973a). Manual activity during speaking-I. Right-handers. Neuropsychologia, 11, 34-50.

Kimura, D. (1973b). Manual activity during speaking-II. Left-handers. Neuropsychologia, 11, 51-55.

Kita, S. (2000). How representational gestures help speaking. In D. McNeill (Ed.), Language and gesture (pp. 162185). Cambridge, England: Cambridge University Press.

Lew, A. R., \& Butterworth, G. (1997). The development of hand-mouth coordination in 2- to 5-month-old infants: Similarities with reaching and grasping. Infant Behavior and Development, 20, 59-69.

Locke, J. L., Bekken, K. E., McMinn-Larson, L., \& Wein, D. (1995). Emergent control of manual and vocal-motor activity in relation to the development of speech. Brain and Language, 51, 498-508.

Lynch, M. P., Oller, D. K., Steffens, M. L., \& Buder, E. H. (1995). Phrasing in prelinguistic vocalizations. Developmental Psychobiology, 28, 3-23.

Lynch, M. P., Oller, D. K., Steffens, M. L., Levine, S. L., Basinger, D. L., \& Umbel, V. (1995). Onset of speech-like vocalizations in infants with Down Syndrome. American Journal on Mental Retardation, 100, 68-86.

MacNeilage, P. F., \& Davis, B. L. (1993). Motor explanations of babbling and early speech patterns. In B. de BoyssonBardies, S. de Schoenen, P. Jusczyk, P. F. MacNeilage, \& J. Morton (Eds.), Developmental neurocognition: Speech and face processing during the first year of life (pp. 341-352). Dordrecht, Netherlands: Kluwer.

MacNeilage, P. F., \& Davis, B. L. (2000). On the origin of internal structure of word forms. Science, 288, 527-531.

MacNeilage, P. F., Davis, B. L., Kinney, A., \& Matyear, C. L. (1999). Origin of serial-output complexity in speech. Psychological Science, 10, 459-460.

Mayberry, R. I., \& Jaques, J. (2000). Gesture production during stuttered speech: Insights into the nature of gesture-speech integration. In D. McNeill (Ed.), Language and gesture (pp. 199-214). Cambridge, England: Cambridge University Press.

McNeill, D. (1992). Hand and mind: What gesture reveals about thought. Chicago: University of Chicago Press.

McNeill, D. (Ed.). (2000). Language and gesture. Cambridge, England: Cambridge University Press.

Meier, R. P., McGarvin, L., Zakia, R. A. E., \& Willerman, R. (1997). Silent mandibular oscillations in vocal babbling. Phonetica, 54, 153-171.

Nathani, S., \& Oller, D. K. (2001). Beyond ba-ba and gu-gu: Challenges and strategies in coding infant vocalizations. Behavior Research Methods, Instruments, and Computers, 33, $321-330$.

Nobe, S. (2000). Where do most spontaneous representational gestures actually occur with respect to speech? In D. McNeill (Ed.), Language and gesture (pp. 186-198). Cambridge, England: Cambridge University Press.

Oller, D. K. (1980). The emergence of speech sounds in infancy. In G. H. Yeni-Komshian, C. A. Ferguson, \& J. Kavanaugh (Eds.), Child phonology: Production (Vol. 1, pp. 93-112). New York: Academic Press. 
Oller, D. K. (2000). The emergence of the speech capacity. Mahwah, NJ: Erlbaum.

Ramsay, D. S. (1984). Onset of duplicated syllable babbling and unimanual handedness in infancy: Evidence for developmental change in hemispheric specialization? Developmental Psychology, 20, 64-71.

Ramsay, D. S. (1985). Fluctuations in unimanual hand preference in infants following onset of duplicated syllable babbling. Developmental Psychology, 21, 318-324.

Rauscher, F., Krauss, R., \& Chen, Y. (1996). Gesture, speech, and lexical access: The role of lexical movements in speech production. Psychological Science, 7, 226-231.

Stark, R. (1980). Stages of speech development in the first year of life. In G. H. Yeni-Komshian, C. A. Ferguson, \& J. Kavanaugh (Eds.), Child phonology: Production (Vol. 1, pp. 73-92). New York: Academic Press.
Stoel-Gammon, C. (1992). Prelinguistic vocal development. In C. Ferguson, L. Menn, \& C. Stoel-Gammon (Eds.), Phonological development (pp. 439-456). Parkton, MD: York Press.

Stoel-Gammon, C. (1998). Role of babbling and phonology in early linguistic development. In A. M. Wetherby, S. F. Warren, \& J. Reichle (Eds.), Transitions in prelinguistic communication (pp. 87-110). Baltimore: Brookes.

Thelen, E. (1979). Rhythmical stereotypies in normal human infants. Animal Behaviour, 27, 699-715.

Thelen, E. (1981a). Rhythmical behavior in infancy: An ethological perspective. Developmental Psychology, 17, 237-257.

Thelen, E. (1981b). Kicking, rocking, and waving: Contextual analyses of rhythmical stereotypies in normal human infants. Animal Behaviour, 29, 3-11. 